\title{
Magnetic Breakdown and Klein Tunneling in a Type-II Weyl Semimetal
}

\author{
T. E. O’Brien, M. Diez, and C. W. J. Beenakker \\ Instituut-Lorentz, Universiteit Leiden, P. O. Box 9506, 2300 RA Leiden, The Netherlands
}

(Received 21 April 2016; published 8 June 2016)

\begin{abstract}
The band structure of a type-II Weyl semimetal has pairs of electron and hole pockets that coexist over a range of energies and touch at a topologically protected conical point. We identify signatures of this Weyl point in the magnetic quantum oscillations of the density of states, observable in thermodynamic properties. Tunneling between the electron and hole pockets in a magnetic field is the momentum space counterpart of Klein tunneling at a $p-n$ junction in real space. This magnetic breakdown happens at a characteristic field strength that vanishes when the Fermi level approaches the Weyl point. The topological distinction between connected and disconnected pairs of type-II Weyl cones can be distinguished by the qualitatively different dependence of the quantum oscillations on the direction of the magnetic field.
\end{abstract}

DOI: 10.1103/PhysRevLett.116.236401

Weyl semimetals provide a condensed matter realization of massless relativistic fermions [1]. Their spectrum features a diabolo-shaped surface in energy-momentum space that separates helical electronlike states (moving in the direction of the momentum) from holelike states (moving opposite to the momentum) [2]. These "Weyl cones" are the three-dimensional analogue of the two-dimensional Dirac cones in graphene. The third spatial dimension provides a topological protection, by which the conical point (Weyl point) cannot be opened up unless two Weyl cones of opposite helicity are brought together in momentum space [3].

Although the Weyl point cannot be locally removed, the cones can be tilted and may even tip over [4-12]. For the relativistic Weyl cone such a distortion is forbidden by particle-hole symmetry, but that is not a fundamental symmetry in condensed matter. While in graphene the high symmetry of the honeycomb lattice keeps the cone upright, strain providing only a weak tilt [13], the tilting can be strong in 3D Weyl semimetals. This leads to a natural division of Weyl cones into two topologically distinct types [9]. In type I the cone is only weakly tilted so that the electronlike states and holelike states occupy separate energy ranges, above or below the Weyl point. In type II the cone has tipped over so that electron and hole states coexist in energy. Many experimental realizations of a typeII Weyl semimetal have recently been reported [14-21].

In a magnetic field the coexisting electron and hole pockets of a type-II Weyl semimetal are coupled by tunneling through the Weyl point (Fig. 1). Here we investigate how this process, a momentum space manifestation of Klein tunneling [22], affects the magnetic quantum oscillations of the density of states (de Haas-van Alphen effect), providing a unique thermodynamic signature of the topologically protected band structure (an alternative to proposed transport signatures [9,23-25]). Because the quantum oscillations are governed by extremal cross sections of the Fermi surface, one might wonder whether some symmetry is required to align the extremal cross section with the Weyl point, so that it becomes observable. Our analysis shows that a magnetic field axis for this alignment exists generically, because of the Möbius strip topology of the projective plane. We first consider Klein tunneling through a single type-II Weyl point, and then turn to pairs of Weyl cones of opposite helicity, which can be combined in topologically distinct ways [8]—with a qualitatively different dependence on the Klein tunneling probability.

To first order in momentum $\boldsymbol{k}$, the Hamiltonian of a Weyl cone has the generic form
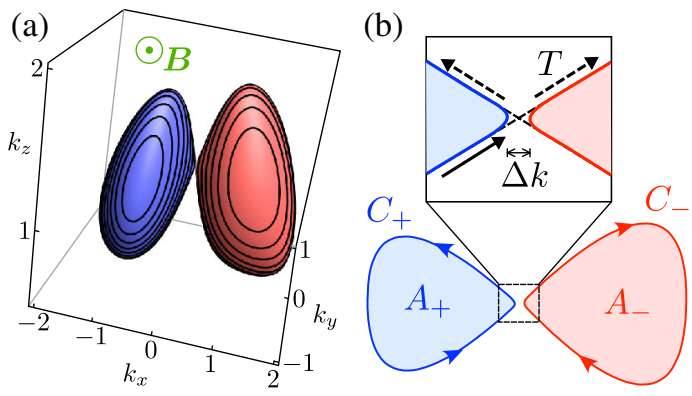

FIG. 1. (a) Fermi surface of a type-II Weyl semimetal, calculated from the model Hamiltonian (1), showing the electron and hole pockets touching at the Weyl point. Equienergy contours in planes perpendicular to the magnetic field $B$ are indicated. The magnetic quantum oscillations have a periodicity in $1 / B$ determined by the contour that encloses an extremal area. (b) Intersection of the Fermi surface with a plane perpendicular to $B$ that passes near the Weyl point. Electron and hole pockets are bounded by a contour $C_{ \pm}$enclosing an area $A_{ \pm}$. The semiclassical orbit of an electron follows the contour in the direction of the arrow. Tunneling between the pockets happens with a probability $T$ that tends to unity when their minimal separation $\Delta k \rightarrow 0$. This magnetic breakdown is a manifestation of Klein tunneling in momentum space. 


$$
H=\sum_{i j} v_{i j} k_{i} \sigma_{j}+a_{\mathrm{tilt}} k_{x} \sigma_{0},
$$

in terms of Pauli matrices $\sigma_{i}, i \in\{x, y, z\}$ (unit matrix $\sigma_{0}$ ). The eigenvalues lie on two hyperboloid sheets $E_{ \pm}$,

$$
E_{ \pm}=a_{\mathrm{tilt}} k_{x} \pm \sqrt{\sum_{i j l} v_{i l} v_{j l} k_{i} k_{j}},
$$

that touch at the Weyl point $\boldsymbol{k}=0$.

For sufficiently small $a_{\text {tilt }}$ the Fermi surface contains either electronlike states in $E_{+}$or holelike states in $E_{-}$, depending on the sign of the Fermi energy relative to the Weyl point. With increasing $a_{\text {tilt }}$ the Weyl cone is tilted in the (arbitrarily chosen) $x$ direction, and when it tips over coexisting electron and hole states appear on the Fermi surface. This is the type-I to type-II Weyl semimetal transition [9].

The hyperboloid dispersion (2) only holds near the Weyl point. In the physical realizations of a type-II Weyl semimetal the Fermi surface closes away from the Weyl point, forming compact electron and hole pockets. A cross section is defined by fixing an axis (unit vector $\hat{\boldsymbol{n}}$ ) and choosing a coordinate $q$ along that axis. The intersection of the Fermi surface with the plane $\hat{\boldsymbol{n}} \cdot \boldsymbol{k}=q$ is an oriented contour $C_{ \pm}(q)$ enclosing the signed area $A_{ \pm}(q)$ (positive for $C_{+}$and negative for $C_{-}$). The contours are the classical momentum-space orbits for a magnetic field $B$ in the $\hat{\boldsymbol{n}}$ direction, the change in orientation between $C_{+}$and $C_{-}$resulting from the sign change of the effective mass in the electron and hole pockets.

Semiclassical quantization of the orbits produces Landau tubes [26], with quantized cross-sectional area

$$
A_{ \pm}(q)=2 \pi(n+\nu) e B / \hbar, \quad n= \pm 1, \pm 2 .
$$

The Maslov index $\nu=1 / 2$ for massive electrons, while $\nu=0$ for massless Weyl fermions [27]. The Landau tubes give rise to oscillations in the density of states periodic in $1 / B$ [28],

$$
\delta \rho / \rho_{0}=\operatorname{Re}\left\{\left[-i A_{ \pm}^{\prime \prime}\left(q_{c}\right)\right]^{-1 / 2} e^{2 \pi i\left(F_{ \pm} / B-\nu\right)}\right\},
$$

with frequency given by the Onsager relation $[29,30]$

$$
F_{ \pm}=(\hbar / 2 \pi e)\left|\mathcal{A}_{ \pm}\right|
$$

The extremal area $\mathcal{A}_{ \pm}=A_{ \pm}\left(q_{c}\right)$ is the area at which the first derivative $d A_{ \pm}(q) / d q=0$. The contour enclosing the extremal area is denoted by $\mathcal{C}_{ \pm}$.

The two sheets $E_{ \pm}$of a type-II Weyl cone are coupled by quantum tunneling. This magnetic-field-induced tunneling between electron and hole pockets is the momentum space counterpart of Klein tunneling at a $p$ - $n$ junction in graphene [31], and can be analyzed along the same lines [32].
The effect of a magnetic field $B$ in, say, the $y$ direction, with vector potential $\boldsymbol{A}=(B z, 0,0)$, is accounted for by the substitution $k_{x} \mapsto k_{x}+e B z$ (setting $\hbar=1$ ). In momentum representation, the Schrödinger equation $H \psi=E \psi$ reads

$$
\begin{gathered}
i U_{0} \frac{\partial \psi}{\partial k_{z}}=U\left(k_{z}\right) \psi, \quad U_{0}=e B\left(\sum_{j} v_{x j} \sigma_{j}+a_{\mathrm{tilt}} \sigma_{0}\right), \\
U\left(k_{z}\right)=E \sigma_{0}-\sum_{i j} v_{i j} k_{i} \sigma_{j}-a_{\mathrm{tilt}} k_{x} \sigma_{0} .
\end{gathered}
$$

For $a_{\mathrm{tilt}}>\left(\sum_{j} v_{x j}^{2}\right)^{1 / 2}$ the matrix $U_{0}$ is positive definite, so that it can be factorized as $U_{0}=V V^{\dagger}$ with invertible $V$ and we may write

$$
i \partial \psi / \partial k_{z}=V^{-1} U\left(k_{z}\right)\left(V^{\dagger}\right)^{-1} \psi \equiv \mathcal{H}\left(k_{z}\right) \psi,
$$

with $\mathcal{H}\left(k_{z}\right)=\mathcal{H}_{0}+\mathcal{H}_{1} k_{z}$. If we interpret $k_{z} \equiv t$ as "time," this looks like a Schrödinger equation for a spin-1/2 particle with time-dependent Hamiltonian $\mathcal{H}(t)$. Because the $t$ dependence of $\mathcal{H}(t)$ is linear, we can use the LandauZener formula for the tunneling probability between the electron and hole pockets [33].

Quite generally, for a two-level system with timedependent Hamiltonian

$$
\mathcal{H}(t)=\left(\begin{array}{cc}
\alpha t+c & \gamma \\
\gamma^{*} & \beta t+c^{\prime}
\end{array}\right)
$$

the Landau-Zener tunnel probability is

$$
T=\exp \left(-2 \pi|\gamma|^{2}|\alpha-\beta|^{-1}\right) .
$$

The matrix (7) is of the form (8) in the basis where $\mathcal{H}_{1}$ is diagonal, so in that basis we can read off the coefficients $\alpha$, $\beta, \gamma$ needed to determine $T$.

For a specific example we consider the Hamiltonian (1) with $v_{i j}=v_{i} \delta_{i j}$, which for $a_{\text {tilt }}>v_{x}$ represents a type-II Weyl cone. We find

$$
\begin{aligned}
T & =\exp \left(-\frac{\pi \hbar}{e B} \frac{v_{x}^{2} E^{2}+v_{y}^{2} k_{y}^{2}\left(a_{\mathrm{tilt}}^{2}-v_{x}^{2}\right)}{v_{z}\left(a_{\mathrm{tilt}}^{2}-v_{x}^{2}\right)^{3 / 2}}\right) \\
& =\exp \left(-\frac{\pi \hbar}{4 e B v_{z}}(\Delta k)^{2}\left(a_{\mathrm{tilt}}^{2}-v_{x}^{2}\right)^{1 / 2}\right)
\end{aligned}
$$

with $\Delta k$ the minimal separation of the contours $C_{+}$and $C_{-}$. This has the general form of the interband tunnel probability in the theory of magnetic breakdown [30,34-36], with a breakdown field $B_{c} \propto(\Delta k)^{2}$. The characteristic feature of Klein tunneling is that the tunnel probability $T \rightarrow 1$ and $B_{c} \rightarrow 0$ at the conical point of the band structure-here a 3D Weyl point and a 2D Dirac point in Ref. [22]. 
To illustrate the effect of Klein tunneling between electron and hole pockets on the magnetic quantum oscillations in the density of states, we consider the model Hamiltonian [37]

$$
\begin{aligned}
H= & \tau_{z}\left(t^{\prime} \sigma_{x} \sin k_{x}+t^{\prime} \sigma_{y} \sin k_{y}\right)+t \tau_{z} \sigma_{0} \sin k_{z} \\
& +\tau_{x} \sigma_{0}\left(\mu-t \cos k_{x}-t \cos k_{y}-t \cos k_{z}\right) \\
& +b \tau_{0} \sigma_{z}+\left[a_{\mathrm{tilt}} \sin k_{x}+\xi\left(1-\cos k_{x}\right)\right] \tau_{0} \sigma_{0} .
\end{aligned}
$$

This is a tight-binding Hamiltonian on a cubic lattice (lattice constant $a_{0}=1$ ), with a spin and orbital degree of freedom on each lattice site (Pauli matrices $\sigma_{i}$ and $\tau_{i}$, respectively). The time-reversal symmetry breaking term $b$ splits the Dirac cone into two Weyl cones separated along the $z$ axis. To produce a type-II Weyl semimetal we have added a tilting term $a_{\text {tilt }}$ and a term $\xi$ that breaks the symmetry between the electron and hole pockets.

As derived in Appendix A of the Supplemental Material [38], near a Weyl point the effective low-energy Hamiltonian has the form (1) with diagonal velocity tensor $v_{i j}=v_{i} \delta_{i j}$ given by

$$
\begin{aligned}
& v_{x}=v_{y}=\frac{(2 t-\mu)^{2}-t^{2}+b^{2}}{2 b(2 t-\mu)} t^{\prime}, \\
& v_{z}=\frac{1}{2 b} \sqrt{\left[(t-\mu)^{2}-b^{2}\right]\left[b^{2}-(3 t-\mu)^{2}\right]} .
\end{aligned}
$$

The Hamiltonian (11) retains a mirror symmetry in the $x-z$ plane (to be removed later on), which implies that for a magnetic field in the $y$ direction the areas $A_{ \pm}\left(k_{y}\right)$ are extremal for $k_{y}=0$. By means of exact diagonalization [39] we have calculated the partial density of states $\rho\left(E, B, k_{y}\right)=\sum_{p} \delta\left[E-E_{p}\left(B, k_{y}\right)\right]$ for $k_{y}=0$, assuming that this gives the dominant contribution to the magnetic quantum oscillations. We choose the gauge $\boldsymbol{A}=(0,0,-B x)$, with a rational flux $B a_{0}^{2}=1 / N \times h / e$ through a unit cell. The lattice has dimensions $N \times N M$ in the $x-z$ plane $(M \gg N \gg 1)$, with periodic boundary conditions in both directions.

Figure 2 shows the energy spectrum as a function of magnetic field and Fig. 3 shows the periodicity of the magnetic oscillations, extracted from a Fourier transform of the density of states. When the Fermi level is far from the Weyl point $E=0$, the electron and hole pockets contribute separately with frequencies $F_{ \pm}$set by the extremal areas $\mathcal{A}_{ \pm}$. The slopes $d F_{ \pm} / d E$ have opposite sign in the two pockets, signifying the opposite sign of the cyclotron effective mass

$$
m_{ \pm}=\frac{\hbar^{2}}{2 \pi} \frac{d}{d E}\left|\mathcal{A}_{ \pm}\right|
$$

Near the Weyl point a low-frequency component appears at the difference $\left|F_{+}-F_{-}\right|$, and the individual

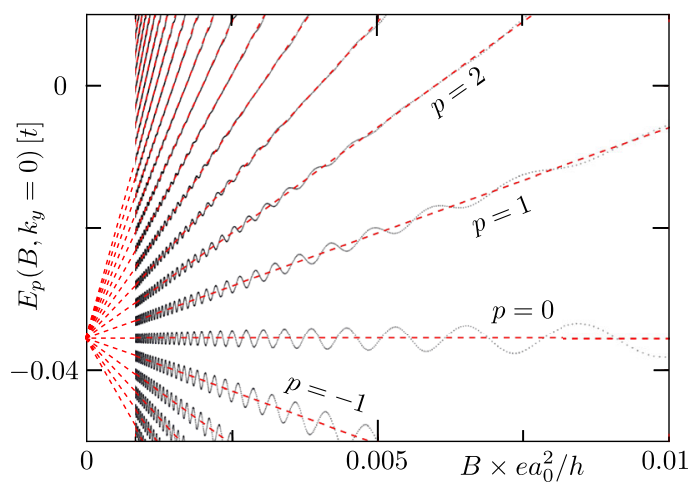

FIG. 2. Energy spectrum at $k_{y}=0$ of the type-II Weyl semimetal with Hamiltonian (11) (parameters $t=1, t^{\prime}=2$, $\left.\mu=3, b=1.2, a_{\text {tilt }}=1.7, \xi=0.08\right)$. The black dotted curves are the exact numerical results, the red dashed lines form the semiclassical Landau fan (15) for tunnel-coupled electron and hole pockets. The individual pockets are responsible for the highfrequency oscillations superimposed on the fan.

high-frequency components $F_{ \pm}$are suppressed. In a semiclassical description, the orbit responsible for the difference frequency is the "figure of eight" orbit formed by joining $C_{+}$to $C_{-}$at the Weyl point [see Fig. 1(b)]. The corresponding effective mass

$$
m_{\Sigma}=\frac{\hbar^{2}}{2 \pi} \frac{d}{d E}\left|\mathcal{A}_{+}+\mathcal{A}_{-}\right|
$$

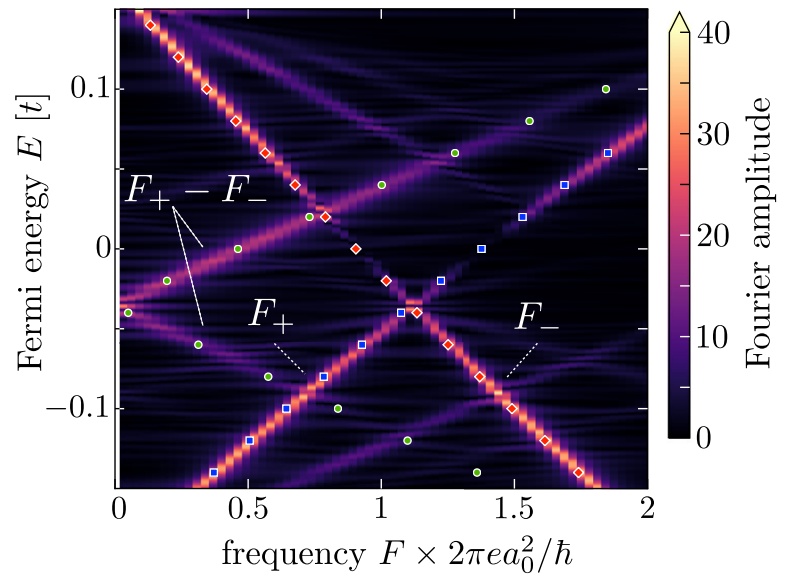

FIG. 3. Fourier amplitudes of the magnetic quantum oscillations. The numerical data for the partial density of states $\rho\left(E, k_{y}=0\right)$ (smoothed with a Gaussian of width $\Gamma=t / 500$ ) are Fourier transformed over the field range $B \lesssim 0.005 \mathrm{~h} / e a_{0}^{2}$ $(200<N<1500)$. The fundamental frequencies from the electron and hole pockets are indicated by $F_{+}$and $F_{-}$, respectively (the first harmonics are also faintly visible). Klein tunneling between the pockets when the Fermi energy approaches the Weyl point $(E=0)$ suppresses these high-frequency oscillations, introducing a new component at the difference frequency $\left|F_{+}-F_{-}\right|$. The colored data points for $F_{ \pm}$are the semiclassical prediction (5) from the extremal areas. 


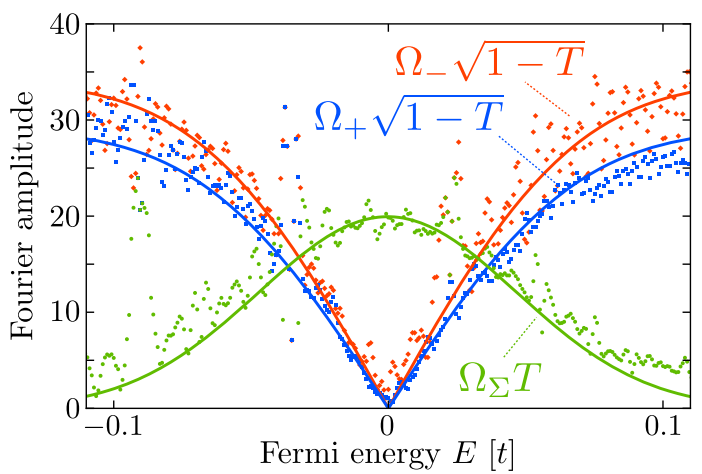

FIG. 4. Energy dependence of the Fourier amplitudes from Fig. 3. The curves are fits to $\Omega_{ \pm} \sqrt{1-T}$ and $\Omega_{\Sigma} T$, with the transmission probability $T(E)$ calculated from Eq. (10) and energy-independent fit parameters $\Omega_{ \pm}, \Omega_{\Sigma}$. When two frequency lines in Fig. 3 cross we cannot reliably determine the individual amplitudes - which explains some of the large scatter in the data points.

governs the Landau fan in Fig. 2,

$$
E_{p}(B)=E_{p}(0)+p \times \hbar e B / m_{\Sigma} .
$$

Notice the absence of a $1 / 2$ offset from the integer $p$, canceled by a Berry phase.

The tunnel probability (10) evaluates for our model parameters to $T(E)=\exp \left[-0.52 N(E / t)^{2}\right]$. The contribution of an orbit to the Fourier amplitude contains a factor $t=\sqrt{T}$ for each transmission through the Weyl point and a factor $r=\sqrt{1-T}$ for each reflection [40]. In Fig. 4 we plot the peak heights of Fig. 3 as a function of energy. The solid lines are fits to $\Omega_{ \pm} \sqrt{1-T(E)}$ and $\Omega_{\Sigma} T(E)$, with energyindependent fit parameters $\Omega_{ \pm}, \Omega_{\Sigma}$. We take for the inverse field strength $N=850$, the midpoint of the interval used in the Fourier transform. A good match to the predicted Gaussian $T(E)$ is obtained.

The above analysis was simplified by the mirror symmetry in the $x-z$ plane, because we could immediately identify the special magnetic field axis for which the extremal contours $\mathcal{C}_{ \pm}$in the electron and hole pockets both touch the Weyl point when $E \rightarrow 0$, allowing for Klein tunneling. One might wonder how restrictive this alignment is-is it possible to find such a special axis in the absence of any symmetry? The answer is yes, as we demonstrate with the help of Fig. 5. At $E=0$ we plot the polar and azimuthal angles $\theta_{ \pm}, \phi_{ \pm}$of the magnetic field axis for which the extremal contour $\mathcal{C}_{ \pm}$touches the Weyl point. Because $(\theta, \phi)$ and $(\pi-\theta, \pi+\phi)$ represent the same axis, we may restrict $\phi$ to the range $[0, \pi]$ - half the usual range for spherical coordinates-identifying the end points $(\theta, 0)$ and $(\pi-\theta, \pi)$. The $(\theta, \phi)$ plane with these "twisted" periodic boundary conditions is the so-called projective plane $\mathbb{P}_{2}$, and has the topology of a Möbius strip.

If the loops $L_{+}=\left(\theta_{+}, \phi_{+}\right)$and $L_{-}=\left(\theta_{-}, \phi_{-}\right)$both wind around the Möbius strip, as they do in Fig. 5, they

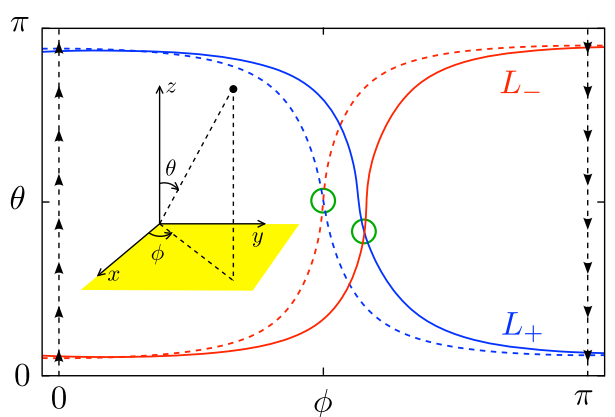

FIG. 5. Magnetic field axis $L_{ \pm}=\left(\theta_{ \pm}, \phi_{ \pm}\right)$for which the extremal contour $\mathcal{C}_{ \pm}$at $E=0$ touches the Weyl point. The dashed curves correspond to the Hamiltonian (11) with the parameters of Fig. 1. For the solid curves we have broken the mirror symmetry by adding the term $V_{0} \tau_{0} \sigma_{0} \sin k_{y}$ with $V_{0}=0.5$. The intersection of $L_{+}$and $L_{-}$(encircled) is the special axis at which Klein tunneling between electron and hole pockets produces magnetic quantum oscillations with the difference frequency $\left|F_{+}-F_{-}\right|$, suppressing both the electron and hole frequencies $F_{ \pm}$. The intersection is protected by the topology of the Möbius strip (indicated by arrows, which show how the edges at $\phi=0, \pi$ should be glued with a twist).

must necessarily intersect because of the twist. The point of intersection is the special axis at which both $\mathcal{C}_{+}$and $\mathcal{C}_{-}$ touch the Weyl point. In Appendix B in the Supplemental Material [38] we show that such noncontractible loops always exist if the Fermi surface is convex, independent of any symmetry requirement.

So far we considered Klein tunneling at a single Weyl point. A second Weyl point of opposite helicity necessarily

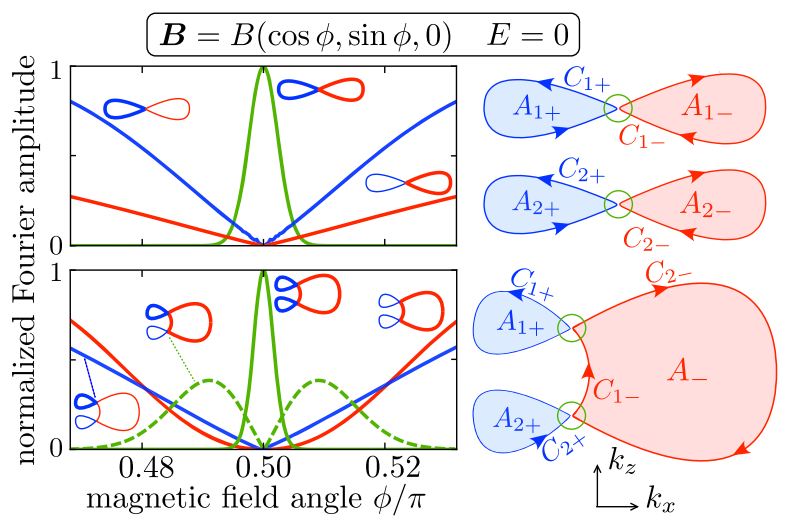

FIG. 6. Left panels: Dependence on the orientation of the magnetic field of the amplitude of the magnetic quantum oscillations (normalized to unit maximal amplitude), for a fixed Fermi energy $E_{F}=0$. Pairs of type-II Weyl points at $E=0$ with disconnected or connected Fermi surfaces are compared. The right panels show a cross section through the electron and hole pockets. For each curve in the left panels the corresponding orbit is indicated. The calculations, detailed in Appendix $\mathrm{C}$ in the Supplemental Material [38], are for the Hamiltonian (11) with parameters $t=1, \mu=3, b=1.2, a_{\text {tilt }}=1.7$ for all panels and $t^{\prime}=2, \xi=0.08$ (top panels); $t^{\prime}=1.7, \xi=0.24$ (bottom panels). 
exists in the Brillouin zone, and this allows for topologically distinct Fermi surfaces [8]. In Fig. 6 we illustrate how Klein tunneling can distinguish connected from disconnected pairs of type-II Weyl cones, by the qualitatively different dependence on the magnetic field orientation.

Experimentally, Klein tunneling through a type-II Weyl point can be detected in measurements of the de Haas-van Alphen effect in the magnetic susceptibility. With sufficient doping $\mathrm{WTe}_{2}$ would produce disconnected type-II Weyl cones near the Fermi energy [9,41], while they are connected in undoped LaAlGe [15]. Klein tunneling is a powerful diagnostic for such topologically distinct Fermi surfaces.

We thank I. Adagideli, P. Baireuther, J. A. Hutasoit, B. Tarasinski, and M. Wimmer for valuable discussions. This research was supported by the Foundation for Fundamental Research on Matter (FOM), the Netherlands Organization for Scientific Research (NWO/OCW), and an ERC Synergy Grant.

[1] H. Weyl, Elektron und gravitation. I, Z. Phys. 56, 330 (1929).

[2] C. Herring, Accidental degeneracy in the energy bands of crystals, Phys. Rev. 52, 365 (1937).

[3] G. E. Volovik, The Universe in a Helium Droplet (Oxford University Press, New York, 2003).

[4] P. Huhtala and G. E. Volovik, Fermionic microstates within the Painlevé-Gullstrand black hole, JETP 94, 853 (2002).

[5] T. Kawarabayashi, Y. Hatsugai, T. Morimoto, and H. Aoki, Generalization of chiral symmetry for tilted Dirac cones, Int. J. Mod. Phys. Conf. Ser. 11, 145 (2012).

[6] M. Trescher, B. Sbierski, P.W. Brouwer, and E. J. Bergholtz, Quantum transport in Dirac materials: Signatures of tilted and anisotropic Dirac and Weyl cones, Phys. Rev. B 91, 115135 (2015).

[7] Y. Sun, S.-C. Wu, M. N. Ali, C. Felser, and B. Yan, Prediction of Weyl semimetal in orthorhombic $\mathrm{MoTe}_{2}$, Phys. Rev. B 92, 161107(R) (2015).

[8] Y. Xu, F. Zhang, and C. Zhang, Structured Weyl Points in Spin-Orbit Coupled Fermionic Superfluids, Phys. Rev. Lett. 115, 265304 (2015).

[9] A. A. Soluyanov, D. Gresch, Z. Wang, Q-S. Wu, M. Troyer, X. Dai, and B.A. Bernevig, Type-II Weyl semimetals, Nature (London) 527, 495 (2015).

[10] G. Autés, D. Gresch, A. A. Soluyanov, M. Troyer, and O. V. Yazyev, Robust type-II Weyl semimetal phase in transition metal diphosphides $\mathrm{XP}_{2}(\mathrm{X}=\mathrm{Mo}, \mathrm{W})$, arXiv:1603.04624.

[11] K. Koepernik, D. Kasinathan, D. V. Efremov, S. Khim, S. Borisenko, B. Büchner, and J. van den Brink, TaIrTe4: A ternary type-II Weyl semimetal, Phys. Rev. B 93, 201101(R) (2016).

[12] L. Muechler, A. Alexandradinata, T. Neupert, and R. Car, Tilted Dirac fermions, arXiv:1604.01398.

[13] M. O. Goerbig, J.-N. Fuchs, G. Montambaux, and F. Piechon, Tilted anisotropic Dirac cones in quinoid-type graphene and $\alpha-(\text { BEDT-TTF })_{2} \mathrm{I}_{3}$, Phys. Rev. B 78, 045415 (2008).

[14] L. Huang, T. M. McCormick, M. Ochi, Z. Zhao, M. Suzuki, R. Arita, Y. Wu, D. Mou, H. Cao, J. Yan, N. Trivedi, and A. Kaminski, Spectroscopic evidence for type II Weyl semimetal state in MoTe2, arXiv:1603.06482.

[15] S.-Y. Xu et al., Discovery of Lorentz-violating Weyl fermion semimetal state in LaAlGe materials, arXiv: 1603.07318.

[16] K. Deng, G. Wan, P. Deng, K. Zhang, S. Ding, E. Wang, M. Yan, H. Huang, H. Zhang, Z. Xu, J. Denlinger, A. Fedorov, H. Yang, W. Duan, H. Yao, Y. Wu, S. Fan, H. Zhang, X. Chen, and S. Zhou, Experimental observation of topological Fermi arcs in type-II Weyl semimetal MoTe2, arXiv:1603.08508.

[17] J. Jiang, Z. K. Liu, Y. Sun, H. F. Yang, R. Rajamathi, Y. P. Qi, L. X. Yang, C. Chen, H. Peng, C.-C. Hwang, S. Z. Sun, S.-K. Mo, I. Vobornik, J. Fujii, S. S. P. Parkin, C. Felser, B. H. Yan, and Y. L. Chen, Observation of the Type-II Weyl semimetal phase in MoTe2, arXiv:1604.00139.

[18] A. Liang et al., Electronic evidence for type II Weyl semimetal state in MoTe2, arXiv:1604.01706.

[19] N. Xu, Z. J. Wang, A. P. Weber, A. Magrez, P. Bugnon, H. Berger, C. E. Matt, J.Z. Ma, B. B. Fu, B. Q. Lv, N. C. Plumb, M. Radovic, E. Pomjakushina, K. Conder, T. Qian, J. H. Dil, J. Mesot, H. Ding, and M. Shi, Discovery of Weyl semimetal state violating Lorentz invariance in MoTe2, arXiv:1604.02116.

[20] C. Wang et al., Spectroscopic evidence of type II Weyl semimetal state in WTe2, arXiv:1604.04218.

[21] Y. Wu, N. H. Jo, D. Mou, L. Huang, S. L. Bud'ko, P. C. Canfield, and A. Kaminski, Observation of Fermi arcs in type-II Weyl semimetal candidate WTe2, arXiv: 1604.05176.

[22] N. Harrison and S. E. Sebastian, Dirac nodal pockets in the antiferromagnetic parent phase of FeAs superconductors, Phys. Rev. B 80, 224512 (2009).

[23] M.-C. Chang and M.-F. Yang, Chiral magnetic effect in the absence of Weyl node, Phys. Rev. B 92, 205201 (2015).

[24] A. A. Zyuzin and R. P. Tiwari, Intrinsic anomalous hall effect in type-II Weyl semimetals, arXiv:1601.00890 [JETP Lett. (to be published)].

[25] Z. Yu, Y. Yao, and S. A. Yang, Unusual magneto-response in type-II Weyl semimetals, arXiv:1604.04030.

[26] N. W. Ashcroft and N. D. Mermin, Solid State Physics (Cengage Learning, Boston, MA, 2011).

[27] C. M. Wang, H.-Z. Lu, and S.-Q. Shen, Anomalous phase shift of quantum oscillation in 3D topological semimetals, arXiv:1604.01681.

[28] For a convex electron or hole pocket one has $\operatorname{sign} A_{ \pm}^{\prime \prime}\left(q_{c}\right)=\mp 1$, so the factor $\left[-i A_{ \pm}^{\prime \prime}\left(q_{c}\right)\right]^{-1 / 2}$ in Eq. (4) contributes a phase shift $\mp \pi / 4$ to the magnetic oscillations. Higher harmonics, faintly visible in Fig. 3, are not included in this formula. We also note that finite temperature effects and the magnetic response of the electron spin are ignored in our general analysis, but would need to be accounted for when comparing with specific experimental data (see Ref. [30]).

[29] L. Onsager, Interpretation of the de Haas-van Alphen effect, Philos. Mag. 43, 1006 (1952). 
[30] D. Shoenberg, Magnetic Oscillations in Metals (Cambridge University Press, Cambridge, England, 1984).

[31] C. W. J. Beenakker, Colloquium: Andreev reflection and Klein tunneling in graphene, Rev. Mod. Phys. 80, 1337 (2008).

[32] R. Nandkishore and L. Levitov, Common-path interference and oscillatory Zener tunneling in bilayer graphene $p-n$ junctions, Proc. Natl. Acad. Sci. U.S.A. 108, 14021 (2011).

[33] L. D. Landau and E. M. Lifshitz, Course of Theoretical Physics (Elsevier, Oxford, 1977), Vol. 3.

[34] E. I. Blount, Bloch electrons in a magnetic field, Phys. Rev. 126, 1636 (1962).

[35] S. Keppeler and R. Winkler, Anomalous MagnetoOscillations and Spin Precession, Phys. Rev. Lett. 88, 046401 (2002).

[36] For $B \lesssim B_{c}$ the Landau-Zener tunnel probability needs to be regularized to account for a logarithmically diverging reflection phase. For an analysis of this subtlety we refer to A. A. Slutskin, Dynamics of conduction electrons under magnetic breakdown conditions, Sov. Phys. JETP 26, 474 (1968); N. V. Vitanov and B. M. Garraway, Landau-Zener model: Effects of finite coupling duration, Phys. Rev. A 53, 4288 (1996); A. Audouarda, J.-Y. Fortin, D. Vignolles, R. B. Lyubovskii, E. I. Zhilyaeva, R. N. Lyubovskaya, and E. Canadell, Onsager phase factor of quantum oscillations in the organic metal $\theta$-(BEDT-TTF $)_{4} \mathrm{CoBr}_{4}\left(\mathrm{C}_{6} \mathrm{H}_{4} \mathrm{Cl}_{2}\right)$, Synth. Met. 171, 51 (2013).

[37] M. M. Vazifeh and M. Franz, Electromagnetic Response of Weyl Semimetals, Phys. Rev. Lett. 111, 027201 (2013). The Hamiltonian (11) differs from that in this reference by the addition of the $a_{\text {tilt }}$ and $\xi$ terms, and also in the replacement of $\tau_{y} \sigma_{0} \sin k_{z}$ by $\tau_{z} \sigma_{0} \sin k_{z}$. This last change was introduced to produce small electron and hole pockets that do not spread out over the entire Brillouin zone.

[38] See Supplemental Material at http://link.aps.org/ supplemental/10.1103/PhysRevLett.116.236401 for appendixes.

[39] To discretize the model Hamiltonian (11) we used the KWANT toolbox: C. W. Groth, M. Wimmer, A. R. Akhmerov, and X. Waintal, Kwant: a software package for quantum transport, New J. Phys. 16, 063065 (2014).

[40] At the special Fermi energy where electron and hole pockets have the same area, additional contributions to the magnetic oscillation amplitude can appear from orbits that go around both pockets without picking up a net phase shift; see J.-Y. Fortin and A. Audouard, Damping of field-induced chemical potential oscillations in ideal two-band compensated metals, Phys. Rev. B 77, 134440 (2008). We do not see this feature in Fig. 4, presumably because our data is too noisy.

[41] Z. Zhu, X. Lin, J. Liu, B. Fauqué, Q. Tao, C. Yang, Y. Shi, and K. Behnia, Quantum Oscillations, Thermoelectric Coefficients, and the Fermi Surface of Semimetallic $\mathrm{WTe}_{2}$, Phys. Rev. Lett. 114, 176601 (2015). This recent experimental study of magnetic quantum oscillations in semimetallic $\mathrm{WTe}_{2}$ reports the sudden appearance of a new frequency above a critical field. Since the new frequency is the sum rather than the difference of the low-field frequencies, it cannot be associated with Klein tunneling through a Weyl point. 